\title{
Examination of Longitudinal AC Breakdown Strength of Dielectric Surfaces as a function of Elastic Modulus
}

\author{
Emre Kantar $^{1}$, Sverre Hvidsten ${ }^{2}$ and Erling Ildstad ${ }^{1}$ \\ ${ }^{1}$ Department of Electric Power Engineering, Norwegian University of Science and Technology \\ ${ }^{2}$ Department of Electric Power Technology, SINTEF Energy Research
}

\begin{abstract}
This paper examines the influence of the elastic modulus of the polymer insulation on the tangential AC breakdown strength (BDS) of polymer interfaces theoretically and experimentally. In the experiments, four different materials with different elastic moduli, namely crosslinked polyethylene (XLPE), cured end product of epoxy resin (EPOXY), polyether ether ketone (PEEK) and silicone rubber $(\mathrm{SiR})$ were employed under various contact pressures. The BDS of each interface increased as the contact pressure was augmented. As the contact pressure became threefold, the interfacial BDS rose by a factor of $2.4,1.7,1.8$, and 1.4 in the case of the PEEK, EPOXY, $\mathrm{XLPE}$ and $\mathrm{SiR}$ interface, in a sequence following the decrease of the elastic modulus. Under the same contact pressure, it was observed that the lower the elastic modulus, the higher the BDS. The employed contact theory also suggested a decreasing BDS as the modulus was augmented; however, the experimental results tended to deviate widely from the estimated results as the pressure was significantly increased.
\end{abstract}

\section{Introduction}

Subsea cable connectors are vital components of oil and gas installations, future offshore wind and wave energy systems. Although materials and production technologies for subsea applications have gained a fair amount of experience over the years, cable connectors and joints where solid-solid interfaces emerge are still considered the weaker parts of complete cable systems [1-3].

One of the main reasons of a solid-solid interface being weaker than its intrinsic material is that an interface contains microscopic imperfections such as cavities (see Fig. 1), protrusions, and contaminants. Such defects reduce the tangential AC electric breakdown strength (BDS) of the interface notably $[1,2]$. Even in cases when the magnitude of the longitudinal electric field is much lower than the dielectric strength of the intrinsic insulation, the imperfections at the interface cause local electric field enhancements. They are, thus, likely to initiate partial discharges (PD), electrical treeing, and a complete flashover might eventually follow [1-3].

Study of insulating materials and BDS of applications for cables and accessories have been covered to a large extent in the literature. Greenwood et al. and Bhusnan reported that total area of contact at an interfacial surface substantially increases in the cases when the elastic modulus is decreased, the contact pressure is augmented, or both $[4,5]$. The interfacial breakdown between two dielectric surfaces was reported to represent one of the principal causes of failure for power cable joints and connectors, in which elastic modulus of the dielectric material plays a key role [3-5]. There is; however, still a lack of knowledge on the correlation between the elastic modulus and the BDS of the interfacial surfaces. Therefore, the primary objective of this paper is to theoretically and experimentally examine the influence of the elastic modulus on the longitudinal AC breakdown strength of dry-assembled solid-solid interfaces under various contact pressures.

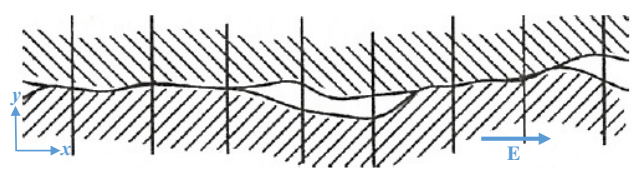

Fig. 1: An illustration of the air-filled cavities at the interface in twodimensional profile. Reference [2] revealed that the voids at the interface are much larger in the tangential direction. They are, thus, referred to as channels [2].

\section{Theory}

In this work, the contact theory $[1,3]$ is employed to model a dry contact between dielectric surfaces. The contact theory suggests that two potential mechanisms are dominating the interfacial breakdown phenomenon [1]. The first hypothesis suggests that the BDS of the contact spots, i.e. the total real area of contact, governs the $\mathrm{BDS}$ of the entire interface. Equation (1) yields the ratio between the total real area of contact $A_{r e}$ (microscopic) and the nominal contact area $A_{a}$ (macroscopic)

$$
A_{r e} \simeq 3.2 A_{a} \frac{p_{a}}{E^{\prime} \sqrt{\sigma / \beta_{m}}}
$$

as a function of the applied contact pressure $p_{a}$, the effective elastic modulus $E^{\prime}$ of two materials in contact, the standard deviation of the asperities' heights $\sigma$ and the mean radius of the asperities' summit $\beta_{m}$ [5]. Thus, the hypothesis renders studying $A_{r e}$ in connection with the interfacial BDS possible under various $E^{\prime}$ and $p_{a}$.

On the other hand, the second hypothesis assumes that the PD in the air-filled microscopic channels, as delineated in Fig. 1, governs the interfacial BDS. Because 
a flashover across a single channel is analogous to the onset of the PD activity at the interface [2] that might eventually evolve to the interfacial breakdown. Following the approach in $[1,3]$, we can estimate the PD inception field strength (PDIE) of a channel using the left-hand side Paschen's curve provided that the average channel size be estimated. The average size of the channels is determined by the following assumptions with regards to the contact theory:

- The summits of the asperities are assumed spherical with a mean radius $\beta_{m}$.

- The asperities have a Gaussian distribution in height about a mean plane in a two-dimensional plane.

- The number of air-filled channels is assumed equal to the number of contact spots at the interface, resulting in one contact spot between two consecutive cavities.

After manipulating the set of formulas provided in [1,3], the correlation between the elastic modulus and the average channel size $d$ reduces to

$$
d=\frac{2\left(E^{\prime} \sqrt{\frac{\sigma}{\beta_{m}}}-3.2 p_{a}\right)^{0.5} \beta_{m}^{0.47} \sigma^{0.41}}{\sqrt{1.21 \pi} E^{\prime 0.06} n^{0.06} p_{a}^{0.44}},
$$

where $n$ is the expected number of the contact spots [1].

Given the fact that $d$ is the average estimated channel size, the PD activity presumably commences at the largest channel(s); however, whether a single or a few large channels can achieve a complete flashover along the interface was not studied. Nor was the duration until the PD activity evolves to a complete flashover examined. With this limited information, we roughly assume that PDIE is linearly proportional to the BDS with the relation of

$$
B D S=\alpha \cdot P D I E,
$$

where $\alpha$ is a numerical coefficient. Both hypotheses will be evaluated in comparison with the measured BDS values in the discussion section.

\section{Experimental Procedure}

\subsection{Mechanical Test Setup}

A simple illustration of the test arrangement with the dimensions of the core components is depicted in Fig. 2. There, two rectangular prism-shaped samples $(55 \mathrm{~mm} \mathrm{x}$ $4 \mathrm{~mm} \times 25 \mathrm{~mm}$ ) were placed on top of each other under dry ambient conditions between two Rogowski-type electrodes, forming a $4 \mathrm{~mm}$-wide interface traversed by the tangentially applied field. For the details of the mechanical setup, readers are advised to refer to [2].

All the breakdown tests were performed with the setup immersed in transformer oil to prevent any external flashover. To avoid ingress of oil at the interface, we applied the contact pressure prior to filling the test chamber with the oil.

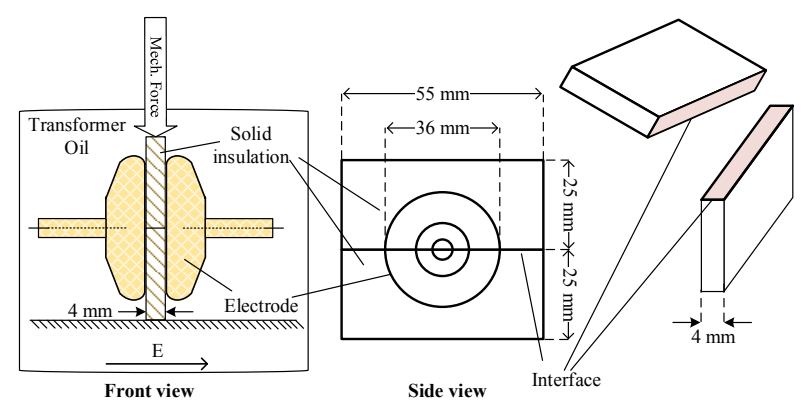

Fig. 2: The simplified sketch of the mechanical test setup.

\subsection{Setup for AC Breakdown Tests}

Fig. 3 shows the whole electrical test setup. A $50 \mathrm{~Hz}$ variac $(0-230 \mathrm{~V})$ was used to energize the primary side of a $100 \mathrm{kV}$ transformer, generating $\mathrm{AC}$ ramp voltage on the secondary winding at the rate of $1 \mathrm{kV} / \mathrm{s}$. A water resistor was employed to limit the breakdown current. Also, a voltage divider was connected in parallel to the test object to transmit secondary voltage information to a $\mathrm{PC}$ via a data acquisition unit.

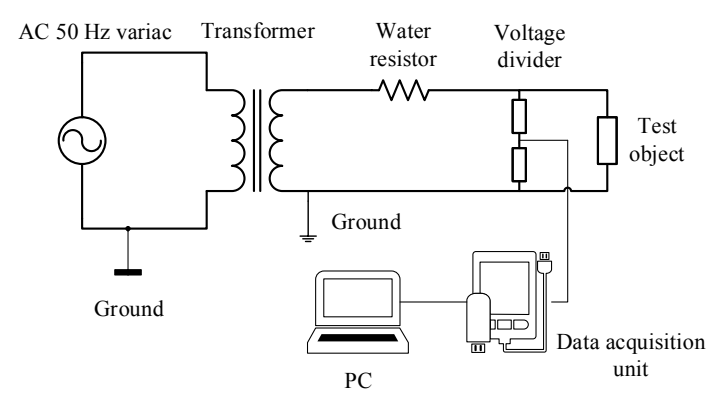

Fig. 3: The sketch of the overall electrical test setup.

\subsection{Preparation of the Samples}

The XLPE and PEEK samples were cut in the aforementioned size from a commercial, XLPE-insulated $145 \mathrm{kV}$ power cable and VESTAKEEP 4000R smooth rod [6], respectively. Whereas, we cast the EPOXY (Casting Resin $X B$ 5950, and Hardener $X B 5951 A P G$ without any fillers [7]) and SiR (Elastosil LR $3003-60 A \& B$ [8]) samples in the laboratory.

The contact surfaces of the samples were polished using STRUERS Abramin table-top, rotating, grinding machine. As shown in [1,2], the specimens were fixed on a steel rotating disk, and a round-SiC sandpaper of the desired grit was placed on the rotating plane. The speed of the rotating plane was set to $150 \mathrm{rpm}$, and the force that presses the steel disk towards sandpaper was fixed to 30 bar during polishing of all the samples, ensuring that surfaces underwent the same procedure. Only grit \#500 type sandpaper was used when polishing the sample surfaces since the influence of various surface roughness on BDS was not studied in this work.

The samples were sanded for $2-3$ minutes with a continuous flow of water to remove any by-products and polymer remnants, and to avoid heating caused by friction. 
Subsequently, the samples were rinsed in tap water and were left to dry in air. Then, the dry samples were cleaned using filtered compressed air before they were washed briefly in isopropanol. Finally, the samples were left to dry again at the room temperature.

\subsection{Elastic Modulus Measurement}

The elastic modulus (Young's modulus) of each material was measured using Lloyd LR5K gauge under tensile testing. The values were determined by the initial slope of the obtained stress-strain curves following the ASTM D 790 standard. Subsequently, the effective elastic modulus $E^{\prime}$ of assembled surfaces were calculated using the following relation

$$
\frac{2}{E^{\prime}}=\frac{\left(1-v_{1}\right)^{2}}{E_{1}}+\frac{\left(1-v_{2}\right)^{2}}{E_{2}},
$$

where $E_{1}, v_{1}$ and $E_{2}, v_{2}$ are the elastic modulus and Poisson's ratio of each surface, respectively [9]. The obtained results are displayed in Table 1.

Table 1: Measured Young's modulus of each sample

\begin{tabular}{cccc}
\hline \hline Interface type & $\begin{array}{c}\text { Young's } \\
\text { Modulus } \\
\boldsymbol{E}[\mathbf{M P a}]\end{array}$ & $\begin{array}{c}\text { Poison's } \\
\text { ratio }\end{array}$ & $\begin{array}{c}\text { Effective } \\
\text { Modulus } \\
\boldsymbol{E}^{\prime}[\mathbf{M P a}]\end{array}$ \\
\hline SiR-SiR & 59 & 0.48 & 109 \\
XLPE-XLPE & 200 & 0.46 & 226 \\
EPOXY-EPOXY & 4425 & 0.38 & 5166 \\
PEEK-PEEK & 7515 & 0.38 & 8808 \\
\hline \hline
\end{tabular}

\subsection{Surface Characterization}

A 3D optical profilometer (Bruker Contour GT-K 3D Optical Microscope) was used to obtain the surface topography of the polished sample surfaces. The assessment area of the profile was $1.26 \mathrm{~mm}$ x $0.95 \mathrm{~mm}$, which was about $5.5 \%$ of the nominal contact area $A_{a}(4 \mathrm{~mm} \times 55 \mathrm{~mm})$. Several scans were performed at different sections to ensure consistency. Surface characterization parameters of $\sigma, \beta_{m}$, and $n$ were then obtained following the procedure in [1] and are the summarized in Table 2.
Table 2: Surface characterization parameters

\begin{tabular}{cccc}
\hline \hline Interface & $\boldsymbol{\sigma}[\boldsymbol{\mu} \mathbf{m}]$ & $\boldsymbol{\beta}_{\boldsymbol{m}}[\boldsymbol{\mu} \mathbf{m}]$ & $\boldsymbol{n}$ \\
\hline SiR & 1.07 & 20.39 & $1.6 \cdot 10^{9}$ \\
XLPE & 2.55 & 6.39 & $2.8 \cdot 10^{9}$ \\
EPOXY & 3.51 & 3.45 & $2.7 \cdot 10^{9}$ \\
PEEK & 2.99 & 1.38 & $7.3 \cdot 10^{9}$ \\
\hline \hline
\end{tabular}

\subsection{Test Procedure and Data Processing}

Initial breakdown tests were performed to identify minimum and maximum forces that the constructed setup permits without oil ingress and deformation of the samples, respectively. The minimum and maximum pressure values together with the intermediate steps determined for each interface are shown in Table 3. The desired force was exerted using weights ranging between $3-75 \mathrm{~kg}$ to press the samples against one another vertically. In each case, the contact pressure was then calculated using $p_{a}=F / A_{a}$, where $F$ is the exerted force in $\mathrm{N}$.

Table 3: Overview of the applied contact pressure values

\begin{tabular}{ccccc}
\hline \hline \multirow{2}{*}{ Interface type } & \multicolumn{4}{c}{ Contact Pressure [bar] } \\
\cline { 2 - 5 } & $\boldsymbol{p}_{\boldsymbol{a} \mathbf{1}}$ & $\boldsymbol{p}_{\boldsymbol{a} \mathbf{2}}$ & $\boldsymbol{p}_{\boldsymbol{a} \mathbf{3}}$ & $\boldsymbol{p}_{\boldsymbol{a} \mathbf{4}}$ \\
\hline SiR-SiR & 1.6 & 1.9 & 2.4 & 2.7 \\
XLPE-XLPE & 5.0 & 8.6 & 11.6 & 16.7 \\
EPOXY-EPOXY & 11.6 & 16.7 & 22.5 & 33.4 \\
PEEK-PEEK & 11.6 & 16.7 & 22.5 & 33.4 \\
\hline
\end{tabular}

For each set of experiments, 8 measurements were taken using a virgin pair of samples. The obtained results were statistically evaluated using the two-parameter Weibull distribution. For further evaluation, the 63.2 percentile value with its $90 \%$ confidence interval was used.

\section{Results}

The experimental data presented in Fig. 4 demonstrate that an increased elastic modulus (i.e. a harder material) results in a reduced BDS. From the minimum contact pressure $\left(p_{a, \min }=p_{a 1}\right)$ to the maximum $\left(p_{a, \max }=\right.$ $\left.p_{a 4}\right)$, the interfacial BDS rose by a factor of $1.4-2.4$ following the decrease of the elastic modulus among the chosen materials. The 63.2 percentile and mean BDS

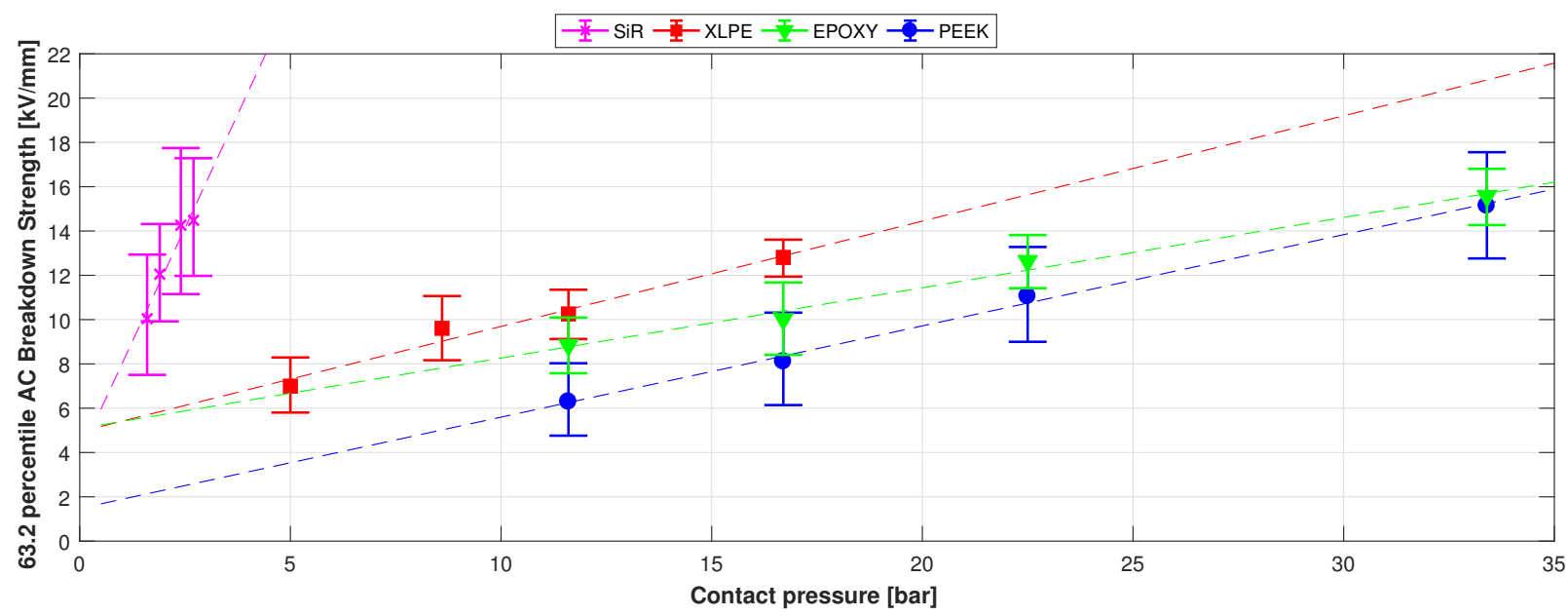

Fig. 4: The 63.2 percentile BDS with the $90 \%$ confidence intervals vs. the contact pressure. The vertical bars feature the $90 \%$ confidence interval of the 63.2 percentile for each case; whereas, the markers point the 63.2 percentile. The dashed lines represent the fitted straight lines to the data points. 


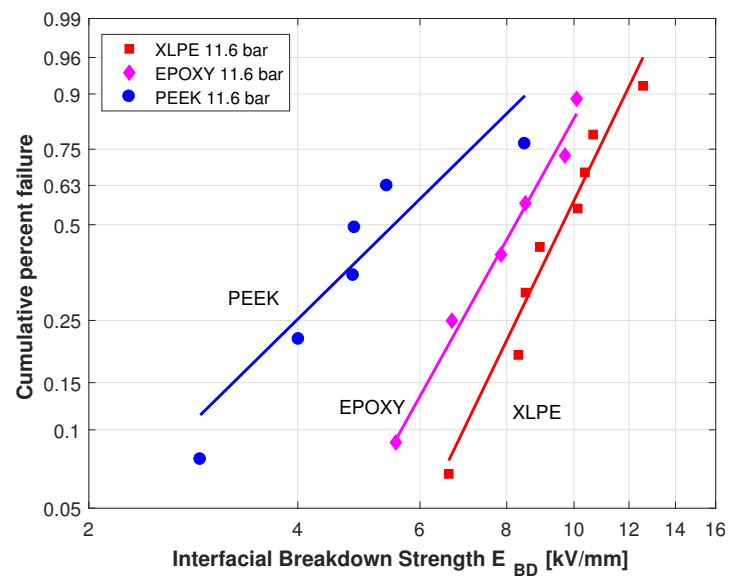

(a)

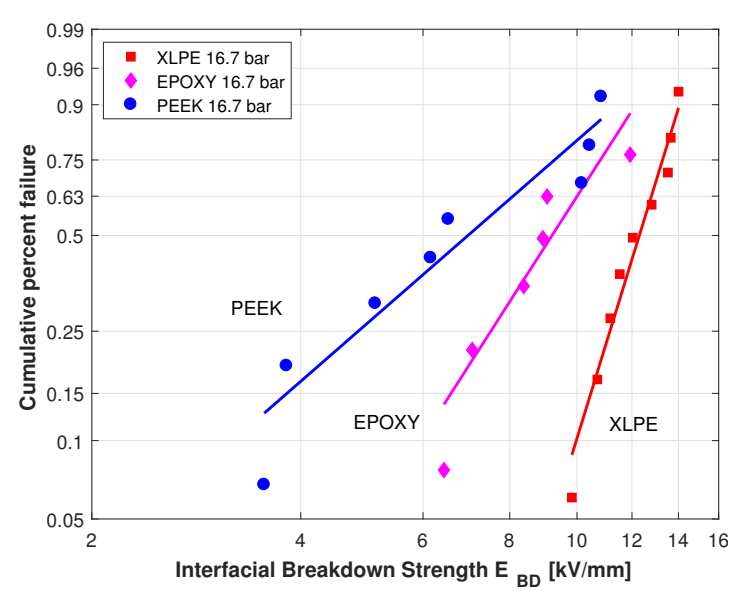

(b)

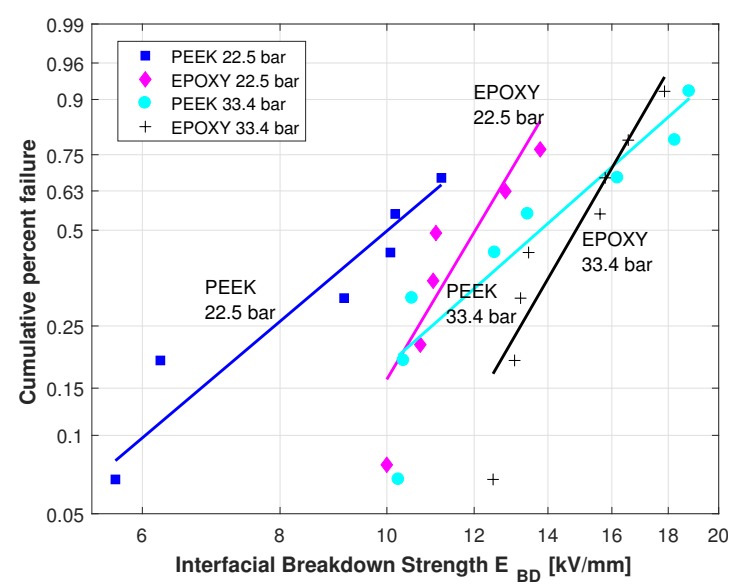

(c)

Fig. 5: The Weibull plot of cumulative percent failure at: (a) 11.6 bar. (b) 16.7 bar. (c) 22.5 and 33.4 bar.

values for each interface are displayed in Table 4 . The dashed lines represent the fitted straight lines to the data points and are extrapolated in the entire pressure range in Fig. 4. The extrapolated SiR data points beyond 5 bar are much higher than the tested dielectric strength of the intrinsic $\mathrm{SiR}(\sim 22 \mathrm{kV} / \mathrm{mm}[8])$, the line is, thus, truncated at $22 \mathrm{kV} / \mathrm{mm}$. Besides, a closer look at the overlapped sections of the bars in Fig. 4 is provided in Fig. 5 in the form of Weibull plots where all the data points are visible.

In addition to the experimentally acquired values, the estimated PDIE values were plotted with the dashed lines in Fig. 6 using the obtained average channel sizes by (2) along with the tabulated data in Table 2. Thus, the variation of $\alpha$ in (3) can be computed by comparing the estimated and experimental data.

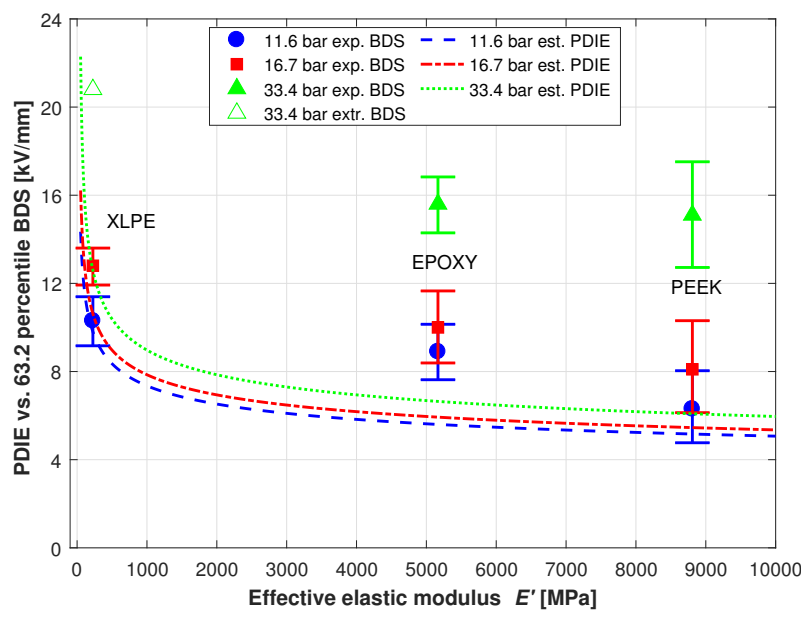

Fig. 6: The estimated PDIE (dashed line) vs. the experimentally obtained 63.2 percentile BDS (marker) with the $90 \%$ confidence interval thereof (bar) as a function of the modulus. For the sake of clarity, only the data at common pressures are covered. [exp: experimental data, extr: extrapolated data, est: estimated data.]

\section{Discussion}

The results presented in Fig. 4 and Table 4 indicated that the rate of change in the $\mathrm{BDS}$ from $p_{a, \min }$ to $p_{a, \max }$ culminates when $E^{\prime}=8808 \mathrm{MPa}$ (PEEK-PEEK); whereas, the lowest gradient is encountered in the case of $E^{\prime}=$ $109 \mathrm{MPa}(\mathrm{SiR}-\mathrm{SiR})$. However, the ratio of $p_{a, \max } / p_{a, \min }$ in the case of SiR-SiR is not as high as those in the other cases. Because the $\mathrm{SiR}$ is a challenging soft material to work with, making it difficult to reduce $p_{a, \text { min }}$ further to prevent oil ingress while $p_{a, \max }$ could not have been increased due to substantial deformation of the samples. Nevertheless, it can still be inferred that the elastic modulus of the material plays a crucial role in the interfacial BDS as follows. In Fig. 4, the 63.2 percentile BDS of the EPOXY and PEEK interfaces can compare with that

Table 4: Overview of the experimental results

\begin{tabular}{|c|c|c|c|c|c|c|c|c|c|c|c|c|}
\hline \multirow{3}{*}{$\begin{array}{c}\text { Interfacial } \\
\text { pressure }\end{array}$} & \multicolumn{12}{|c|}{ Breakdown Strength [kV/mm] } \\
\hline & \multicolumn{3}{|c|}{ SiR-SiR } & \multicolumn{3}{|c|}{ XLPE-XLPE } & \multicolumn{3}{|c|}{ EPOXY-EPOXY } & \multicolumn{3}{|c|}{ PEEK-PEEK } \\
\hline & $p_{a}[\mathrm{bar}]$ & $63 \%$ & Mean & $p_{a}[\mathrm{bar}]$ & $63 \%$ & Mean & $p_{a}[\mathrm{bar}]$ & $63 \%$ & Mean & $p_{a}[\mathrm{bar}]$ & $63 \%$ & Mean \\
\hline$p_{a 2}$ & 1.9 & 12.1 & 9.7 & 8.6 & 9.6 & 8.3 & 16.7 & 10.0 & 8.6 & 16.7 & 8.1 & 7.1 \\
\hline$p_{a 3}$ & 2.4 & 14.3 & 10.8 & 11.6 & 10.3 & 9.5 & 22.5 & 12.6 & 11.6 & 22.5 & 11.1 & 8.8 \\
\hline
\end{tabular}


of the SiR interface only at contact pressures at least ten times as high. It is interesting to observe that materials with low modulus such as the SiR can achieve such high BDS values even at low contact pressures.

As (1)-(2) reveals, the elastic modulus affects both the size of channels and the total area of contact at the interface. In the case of a material with low elastic modulus, increasing the contact pressure will apparently increase the total real area of contact $A_{r e}$ further than it does in the event of a material with high elastic modulus. That, in turn, yields shorter channels as (2) suggests, resulting in a higher PDIE and hence a higher BDS in accordance with (3). With the increase in pressure, overlapped parts of the bars in Fig. 4 at the same pressure becomes larger particularly in the case of the EPOXY and PEEK interfaces, indicating that increase in modulus renders the average channel sizes comparable.

The experimentally obtained BDS values start to deviate considerably from the estimated PDIE values in Fig. 6 as the pressure is increased. Thus, much higher $\alpha$ coefficients arise as the pressure is raised. For instance, the $\alpha$ varies from 1.1 to 1.6 at 11.6 bar; whereas, the range at 33.4 bar becomes $1.7-2.5$ as the modulus is augmented. It can, then, be argued that the interfacial breakdown phenomenon is not directly governed by the air-filled channels at high pressures; in other words, the second hypothesis starts not to hold true as the pressure is increased. There are two plausible explanations as follows: First, the increased contact pressure probably renders the channels much shorter in practice than the model predicts. Therefore, the model estimating the channel sizes should be improved. Secondly, as the first hypothesis suggests, the interfacial BDS might be dominated by the breakdown of the total area of contact at high pressures. The intrinsic insulation is reported to have a nominal BDS range of $22-42,20-24$ and $16-21 \mathrm{kV} / \mathrm{mm}$ for XLPE, EPOXY to PEEK, respectively by the manufacturer data sheets $[3,6,7]$. On that account, the experimental BDS data in Fig. 6 at 33.4 bar are closer to those of the intrinsic materials, favoring the first hypothesis over the second.

Despite the probable coexistence of minuscule channels-whose BDS values are rather high-and contact spots, the intrinsic BDS values are almost reached especially in the cases of EPOXY and PEEK at 33.4 bar. Nevertheless, both the mechanisms are likely to take place simultaneously in real-life. Depending on the contact pressure and the elastic modulus, either of mechanisms prevails over the other.

\section{Conclusion}

- The experiments show that the BDS increases with the increased contact pressure in all cases irrespective of the elastic modulus of the insulation.

- The experimental results indicate that the lower the elastic modulus, the higher the BDS, where a much higher BDS is achieved using softer materials with low elastic modulus.

- The theoretical model suggests that the channel size and hence the PDIE decreases as the elastic modulus is reduced.

- When considering the breakdown of the interfaces, both the breakdown of air-filled channels and real area of contact are essential, since either of them dominates the interfacial BDS, depending strongly on the contact pressure and the modulus.

- To improve the theoretical model and to correlate the interfacial BDS with the elastic modulus in a clearer way, further experiments on the PDIE is essential.

\section{Acknowledgment}

This work is part of the project "High Voltage Subsea Connections (SUBCONN)" funded by the Research Council of Norway (project no. 228344/E30) and the industrial partners: ABB AS, Aker Solutions AS, Deutsch Offshore, Chevron Norge AS, Det Norske Oljeselskap ASA, Nexans Norway AS, Shell Technology Norway AS and Statoil Petroleum AS.

\section{References}

[1] E. Kantar and E. Ildstad, "Modeling longitudinal breakdown strength of solid-solid interfaces using contact theory," in 2016 IEEE Int. Conf. on Dielec. (ICD), vol. 1, July 2016, pp. 398-401.

[2] E. Kantar, S. Hvidsten, F. Mauseth, and E. Ildstad, "Longitudinal AC breakdown voltage of XLPEXLPE interfaces considering surface roughness and pressure," IEEE Trans. Dielectr. Electr. Insul., vol. TBA, no. TBA, 2017.

[3] S. M. Hasheminezhad, "Tangential electric breakdown strength and PD inception voltage of solidsolid interface," Ph.D. dissertation, Norwegian University of Science and Technology, 2016.

[4] J. Greenwood and J. Williamson, "Contact of nominally flat surfaces," in Proceedings of the Royal Society of London A: Mathematical, Physical and Engineering Sciences, vol. 295, no. 1442. The Royal Society, 1966, pp. 300-319.

[5] B. Bhushan, "Analysis of the real area of contact between a polymeric magnetic medium and a rigid surface," Journal of Tribology, vol. 106, no. 1, pp. 2634, 1984.

[6] "PI-VESTAKEEP-4000G-EN data sheet," Evonik Industries AG, Essen, Germany.

[7] "Casting Resin XB 5950 / Hardener XB 5951 APG data sheet," Huntsman Int. LLC, The Woodlands, Texas.

[8] "ELASTOSIL@ LR 3003/60 A/B data sheet," Wacker Chemie AG, Munich, Germany.

[9] A. Almqvist, "On the effects of surface roughness in lubrication," Ph.D. dissertation, Luleå tekniska universitet, 2006. 